\title{
Riesz transforms in statistical signal processing and their applications to speckle metrology: a review
}

Wang, Wei; Zhang, Shun; Ma, Ning; Hanson, Steen Grüner; Takeda, Mitsuo

Published in:

Proceedings of SPIE

Link to article, DOI:

$10.1117 / 12.2081318$

Publication date:

2015

Document Version

Publisher's PDF, also known as Version of record

Link back to DTU Orbit

Citation (APA):

Wang, W., Zhang, S., Ma, N., Hanson, S. G., \& Takeda, M. (2015). Riesz transforms in statistical signal processing and their applications to speckle metrology: a review. In Proceedings of SPIE (Vol. 9449). [944904] SPIE - International Society for Optical Engineering. Proceedings of SPIE - The International Society for Optical Engineering https://doi.org/10.1117/12.2081318

\section{General rights}

Copyright and moral rights for the publications made accessible in the public portal are retained by the authors and/or other copyright owners and it is a condition of accessing publications that users recognise and abide by the legal requirements associated with these rights.

- Users may download and print one copy of any publication from the public portal for the purpose of private study or research.

- You may not further distribute the material or use it for any profit-making activity or commercial gain

- You may freely distribute the URL identifying the publication in the public portal 


\title{
Riesz transforms in statistical signal processing and their applications to speckle metrology: a review
}

\author{
Wei Wang ${ }^{* a}$, Shun Zhang ${ }^{\mathrm{a}}$, Ning Ma ${ }^{\mathrm{a}}$, Steen G. Hanson ${ }^{\mathrm{b}}$, and Mitsuo Takeda ${ }^{\mathrm{c}}$ \\ ${ }^{a}$ Institute of Photonics and Quantum Sciences, School of Engineering and Physical Sciences, \\ Heriot-Watt University, Edinburgh, EH14 4AS, United Kingdom \\ ${ }^{\mathrm{b}}$ DTU Fotonik, Department of Photonics Engineering \\ Technical University of Denmark, Dk-4000 Roskilde, Denmark \\ ${ }^{\mathrm{c}}$ Center for Optical Research and Education, Utsunomiya University, \\ 7-1-2, Yoto, Utsunomiya, Tochigi 321-8585, Japan
}

\begin{abstract}
In this paper, a high-dimensional statistical signal processing is revisited with the aim of introducing the concept of vector signal representation derived from the Riesz transforms, which are the natural extension and generalization of the one-dimensional Hilbert transform. Under the new concepts of vector correlations proposed recently, the statistical properties of the vector signal representation for random signal are presented and some applications to speckle metrology developed recently are reviewed to demonstrate the unique capability of Riesz transforms.
\end{abstract}

Keywords: Riesz transform, speckles, vector correlations, optical vortex metrology, stochastic process

\section{INTRODUCTION}

In physics and engineering, it's common practice and usual treatment to represent a real-valued signal by its related complex-valued signal. The use of the associated complex signal simplifies calculations, especially when averages of quadratic quantities in the field variable have been involved. Meanwhile, the introduced instantaneous frequency, envelop and phase in this complex signal also provide more convenient means for analyzing, processing and understanding the available information from the original real-valued signal. Since the introduction of the analytic signal built from the Hilbert transform for a real one-dimensional (1-D) signal by Gabor in $1946^{1}$, the complex signal representation has become an important tool in 1-D signal processing. It is well-known that an analytic signal consists of two parts: the real part is the original signal and the imaginary part is the Hilbert transform of the real part ${ }^{24}$. Although the concept of analytic signal underpins many aspects of modern 1-D signal processing, the Hilbert transform has restricted its application of the analytic signal concept to multiple dimensions due to its inherent preferred directions ${ }^{5-8}$. In the areas of optics, image analysis and visual perception, many attempts have been made to extend the Hilbert transform beyond one dimension naturally without implying a preferred direction ${ }^{5-9}$. Among all these attempts with proposed filters and generated signals, the signal obtained from Riesz transform has become the most promising one as the attempt for the natural extension of concept of 1-D analytic signal $5,8,10$. Although Riesz transforms have found successes in many applications such as fringe analysis ${ }^{8}$, pattern recognition ${ }^{5,7}$ and optical metrology ${ }^{11-15}$, little work has been done to apply such transforms to statistically analyze random signal like speckle patterns. No knowledge has been obtained about the statistical properties of the associated signals when the Riesz transform is applied.

In this paper, we will review the Riesz transforms in statistical signal processing and some applications to speckle metrology. Through the application of Riesz Transform to a 2-D speckle pattern as a typical random signal, the corresponding vector representation has been established in Cartesian and spherical coordinate systems as the natural generalization of 1-D analytic signal. Some correlation properties associated with the vector representation of a real-valued 2-D speckle pattern have been presented, which share the formal analogy to the statistics of the 1-D analytic signal and may serve as a mathematical foundation for the statistical analysis of 2-D signal $^{17}$. Under the framework of the vector signal representation generated by Riesz transforms, the principle and applications of several techniques, such as Optical Vortex Metrology ${ }^{11-14}$ and Pseduo-Stokes vector correlation ${ }^{15-16}$, which we proposed recently for displacement and flow measurements will be revisited to demonstrate the unique capability of Riesz transform.

*w.wang@hw.ac.uk Tel: +44 (0) 131451 3141; Fax: +44 (0) 1314513129

The International Conference on Photonics and Optical Engineering (icPOE 2014),

edited by Ailing Tian, Anand Asundi, Weiguo Liu, Chunmin Zhang, Proc. of SPIE Vol. 9449, 944904

(C) 2015 SPIE · CCC code: 0277-786X/15/\$18 - doi: 10.1117/12.2081318 


\section{VECTORIAL RANDOM PROCESS DERIVED FROM ANALYTIC SIGNAL}

\subsection{Representation of a 2-D real-valued signal by a vector signal}

In 1-D signal processing, Hilbert transform and its associated analytic signal play critical roles and provide mathematical basis to describe statistical signal. Many interesting properties of analytic signal and its statistical behaviors as 1-D complex random process have been discussed and proposed ${ }^{2-4}$. To extend this important concept, Riesz transform has been introduced as a natural generalization to 2-D signal processing with its resulting 3-D vector representation ${ }^{5,7-8}$. Consider a 2-D real-valued function $f(x, y)$, which might represent an intensity fluctuation of an image at point $(x, y)$. By a similar procedure used in monogenic signal ${ }^{5}$, the vector representation in space domain can be introduced as

$$
\vec{A}_{f}(x, y)=\left[k^{(2)}(x, y) e_{1}-k^{(1)}(x, y) e_{2}+\delta(x, y) e_{3}\right] * f(x, y),
$$

where $\hat{e}_{l}(l=1,2,3)$ denote the orthogonal unit vectors, and asterisk $*$ here means the convolution, while $k^{(1)}(x, y)$ and $k^{(2)}(x, y)$ are 2-D Riesz transform kernels defined as

$$
k(x, y)=\left\{\begin{array}{l}
k^{(1)}(x, y)=x /\left[2 \pi\left(x^{2}+y^{2}\right)^{3 / 2}\right] \\
k^{(2)}(x, y)=y /\left[2 \pi\left(x^{2}+y^{2}\right)^{3 / 2}\right]
\end{array}\right.
$$

with their Fourier transforms

$$
\mathfrak{I}\{k(x, y)\}=\left\{\begin{array}{l}
-j \omega_{x} / \sqrt{\omega_{x}^{2}+\omega_{y}^{2}} \\
-j \omega_{y} / \sqrt{\omega_{x}^{2}+\omega_{y}^{2}},
\end{array}\right.
$$

From the equation above, the degeneration expression of Riesz kernel in frequency domain to 1-D becomes $-j \omega /|\vec{\omega}|=-j \operatorname{sgn}(\omega)$, where $\operatorname{sgn}(\omega)$ is the signum function:

$$
\operatorname{sgn}(\omega)=\left\{\begin{array}{cc}
+1 & \omega>0 \\
0 & \omega=0 \\
-1 & \omega<0
\end{array}\right.
$$

Thus, the Hilbert transform denoted by symbol $\mathscr{C}\{\cdots\}$ takes the form $\mathscr{K}\{f(x)\}=-j \operatorname{sgn}(\omega) \mathfrak{I}\{f(x)\}$, as a 1-D special case of Riesz transform in frequency domain. Based on the definition in space domain as shown in Eq. (1), the construction of the vector representation $\vec{A}_{f}$ from the 2-D real-valued signal $f(x, y)$ in frequency domain can thus be illustrated diagrammatically in Fig. 1.

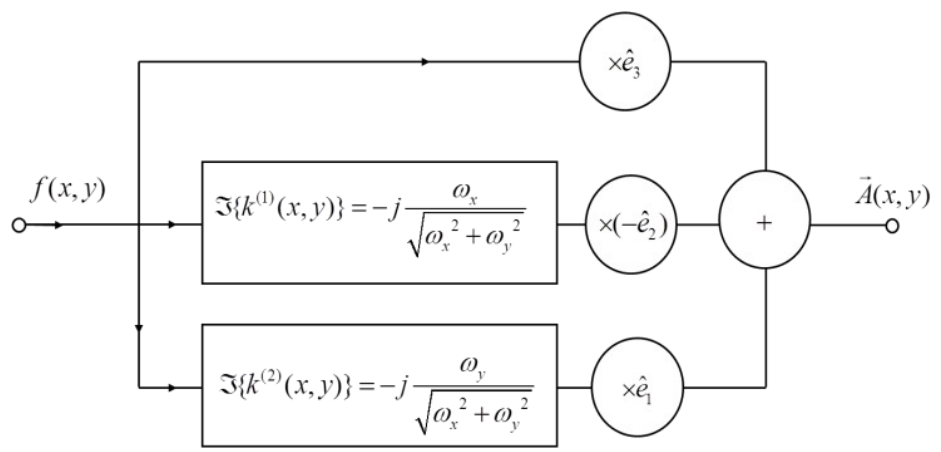

Figure 1. Construction of a vector representation from a real-valued 2-D signal 


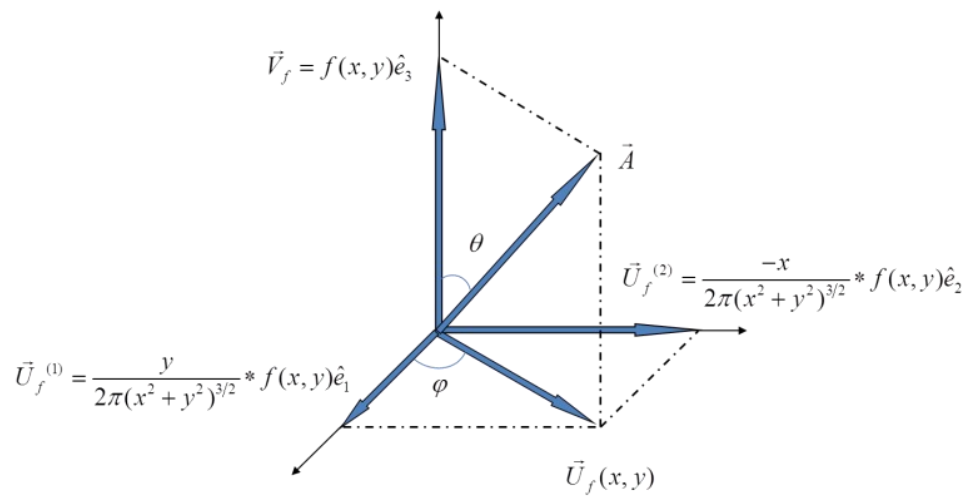

Figure 2. Vector signal representation in the Cartesian and spherical coordinates

Figure 2 is a geometrical representation of $\vec{A}_{f}$ in Cartesian and spherical coordinates with the polar angle $\theta$ and azimuthal angle $\varphi$ indicating local phase and local fringe orientation ${ }^{7,8}$. The vector representation $\vec{A}_{f}$ could also be expressed as $\quad \vec{A}_{f}(x, y)=\vec{U}_{f}(x, y)+\vec{V}_{f}(x, y) \quad$ with $\quad \vec{U}_{f}(x, y)=\left[k^{(2)} * f\right] e_{1}-\left[k^{(1)} * f\right] e_{2}=U_{f}^{(1)} e_{1}+U_{f}^{(2)} e_{2} \quad$ and $\vec{V}_{f}(x, y)=f(x, y) \hat{e}_{3}$. As we will see in the next section, $\vec{V}_{f}$ and $\vec{U}_{f}$ have the counterparts in the 1-D complex analytic signal as real and image parts.

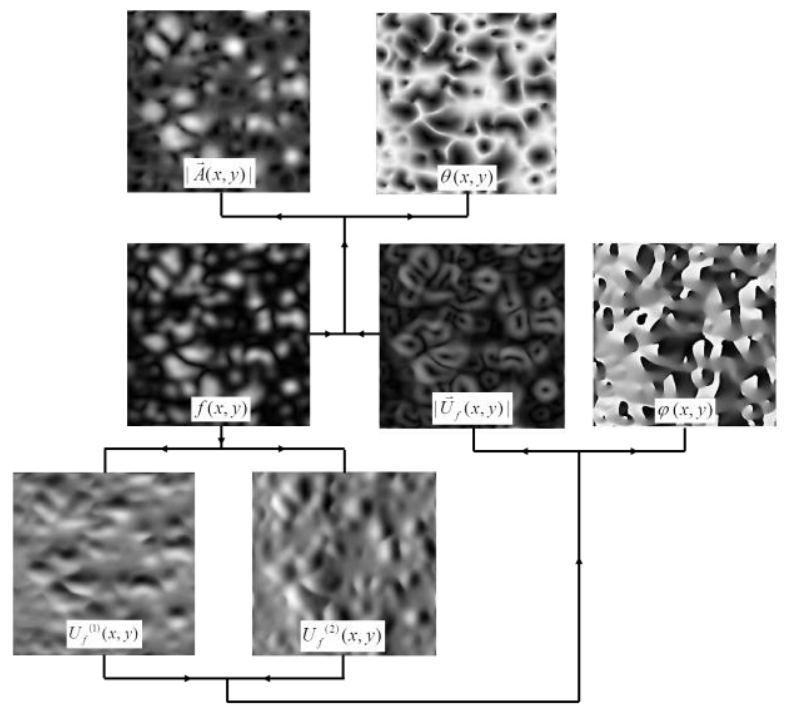

Figure 3. Sample image of a vector representation for 2-D speckle pattern

Figure 3 shows an example of the vector representation of a speckle as a typical real 2-D signal generated from the Riesz transform, where $|\cdots|$ is modulus of real vector. In this example, the image size of 450 pixels by 450 pixels has been chosen as the truncated speckle pattern with its average speckle width of 45 pixels.

\subsection{Statistical properties of the vector signal representation for speckle pattern}

In stochastic processing, especially in the speckle signal analysis, the correlation functions play great roles ${ }^{34}$. To reveal statistical relationship between two random vector representations introduced above, we accordingly give the definition of vector correlations as new mathematical tools for vector calculus. Combining the traditional correlation operation and vector product, the inner and the outer correlations are defined as follows. Given two vector functions $\vec{f}$ and $\vec{g}$, which may be sample functions of vector random processes, the inner correlation function between $\vec{f}$ and $\vec{g}$ is defined by 


$$
\vec{f}(x, y) \oplus \vec{g}(x, y)=\sum_{m=1}^{3}\left[f^{(m)^{*}}(-x,-y) * g^{(m)}(x, y)\right]=\sum_{m=1}^{3} \Gamma_{f, g}^{(m, m)}(x, y),
$$

At the same time, the outer correlation denoted by $\otimes$ can be written as

$$
\vec{f}(x, y) \otimes \vec{g}(x, y)=\Gamma_{f, g}^{(2,3)}(x, y) e_{1}+\Gamma_{f, g}^{(3,1)}(x, y) e_{2}+\Gamma_{f, g}^{(1,2)}(x, y) e_{3}-\Gamma_{f, g}^{(3,2)}(x, y) e_{1}-\Gamma_{f, g}^{(1,3)}(x, y) e_{2}-\Gamma_{f, g}^{(2,3)}(x, y) e_{3} .
$$

From the vector representation of a real-valued 2-D signal by using Riesz transform introduced above, we now start to establish many interesting relations involving correlations of original speckle patterns and the associated vector representations. Let $f(x, y)$ represent the wide-sense stationary original real-valued 2-D speckle signal with zero mean and let $\vec{A}_{f}$ be its vector representation with two components $\vec{V}_{f}$ and $\vec{U}_{f}$. For $\vec{A}_{f}$, the correlation properties between its components are of general interest. Let us consider the inner auto-correlation functions of $\vec{U}_{f}$ and $\vec{V}_{f}$ first. From definition in Eq. (5), we have:

$$
\vec{U}_{f}(x, y) \oplus \vec{U}_{f}(x, y)=-\left[k^{(2)}(x, y) * k^{(2)}(x, y)+k^{(1)}(x, y) * k^{(1)}(x, y)\right] * \Gamma_{f, f}(x, y) .
$$

In derivation of Eq. (7), we have made use of the commutative property of convolution ${ }^{18}$. Note the fact that $k^{(2)} * k^{(2)}+k^{(1)} * k^{(1)}=-\delta(x, y)$, we have

$$
\vec{U}_{f}(x, y) \oplus \vec{U}_{f}(x, y)=\Gamma_{f, f}(x, y)=\vec{V}_{f}(x, y) \oplus \vec{V}_{f}(x, y) .
$$

We notice that Eq. (8) provides the relationship between the inner correlations of vector components of $\vec{A}_{f}$, just as the property for the complex analytic signal where the auto-correlation of real part is equal to that of the imaginary part. For the outer correlation between $\vec{U}_{f}$ and $\vec{V}_{f}$, we use its definition in Eq.(6) and the associative property of convolution ${ }^{18}$. The result is

$$
\vec{U}_{f}(x, y) \otimes \vec{V}_{f}(x, y)=\vec{V}_{f}(x, y) \otimes \vec{U}_{f}(x, y)=\left[k^{(1)}(x, y) e_{1}+k^{(2)}(x, y) e_{2}\right] * \Gamma_{f, f}(x, y) .
$$

From Eq. (9), we notice that the outer correlation between $\vec{U}_{f}$ and $\vec{V}_{f}$ can be obtained by Riesz transform of the original autocorrelation $\Gamma_{f, f}(x, y)$. Since the integrals in convolution are equal to 0 , if it is odd with respect to the integral variables, it is straightforward to derive the following basic property as a special case for outer cross correlation: $\left.\vec{U}_{f} \otimes \vec{V}_{f}\right|_{x, y=0}=\left.\vec{V}_{f} \otimes \vec{U}_{f}\right|_{x, y=0}=0$.

For future applications, it is convenient to give the inner and outer correlation of a vector representation $\vec{A}_{f}$. By expanding the vector signal $\vec{A}_{f}$ in terms of its components, we can readily show that

$$
\vec{A}_{f}(x, y) \oplus \vec{A}_{f}(x, y)=\vec{U}_{f}(x, y) \oplus \vec{U}_{f}(x, y)+\vec{V}_{f}(x, y) \oplus \vec{V}_{f}(x, y)=2 \Gamma_{f, f}(x, y) .
$$

Thus the inner auto-correlation of the vector signal representation is just twice the auto-correlation function of original real-valued speckle pattern. Similarly, using Eq. (6) and Eq. (9), we see directly that

$$
\vec{A}_{f}(x, y) \otimes \vec{A}_{f}(x, y)=2 \vec{U}_{f}(x, y) \otimes \vec{V}_{f}(x, y)=2\left(k^{(1)} e_{1}+k^{(2)} e_{2}\right) * \Gamma_{f, f}(x, y) .
$$

Once again, we notice the Riesz transform relation between outer-correlation of $\vec{A}_{f}$ and the original auto-correlation $\Gamma_{f, f}(x, y)$. Finally, we consider the inner and outer cross-correlation functions of two jointly wide-sense stationary vector representations $\vec{A}_{f}$ and $\vec{A}_{g}$ for two speckle patterns. With the notation $\vec{A}_{g}(x, y)=\vec{U}_{g}(x, y)+\vec{V}_{g}(x, y)$, a direct substitution into Eq. (5) yields

$$
\vec{A}_{f}(x, y) \oplus \vec{A}_{g}(x, y)=2 \Gamma_{f, g}(x, y) .
$$

As shown in Eq. (12), the inner cross-correlation functions between $\vec{A}_{f}$ and $\vec{A}_{g}$ is twice of the cross-correlation function $\Gamma_{f, g}$. By a similar process used in Eq. (11), we have

$$
\begin{gathered}
\vec{U}_{f}(x, y) \otimes \vec{U}_{g}(x, y)=0, \\
\vec{V}_{f}(x, y) \otimes \vec{U}_{g}(x, y)=\vec{U}_{g}(x, y) \otimes \vec{V}_{f}(x, y)=\left[k^{(1)} e_{1}+k^{(2)} e_{2}\right] * \Gamma_{f, g} . \\
\vec{V}_{f}(x, y) \otimes \vec{V}_{g}(x, y)=0 .
\end{gathered}
$$

Proceeding directly, we can readily simplify the outer cross-correlation as

$$
\vec{A}_{f}(x, y) \otimes \vec{A}_{g}(x, y)=2\left(k^{(1)} e_{1}+k^{(2)} e_{2}\right) * \Gamma_{f, g} .
$$


Equation (14) implies that the outer correlation between $\vec{A}_{f}$ and $\vec{A}_{g}$ could be derived from the Riesz transform of the cross-correlation function $\Gamma_{f, g}$. With the help of Eq. (12) and Eq. (14), the inner and outer auto-correlation functions in Eq. (10) and Eq. (11) can be treated as special cases of corresponding cross-correlations. Eq. (12) and (14) reveal the relationships between the cross-correlation functions of the original speckle signals and the associated vector representations.

\section{SOME APPLICATIONS OF RIESZ TRANSFORMS TO SPECKLE METROLOGY}

\subsection{Optical vortex metrology}

Just as a random speckle intensity pattern imprints marks on a coherently illuminated object surface, randomly distributed singularities in the map of azimuthal angle $\varphi$ of the speckle-like pattern imprint unique marks on the object surface. We make use of the information about the locations of these singularities before and after the displacement. To do this, we need to identify the corresponding singularities between the pre- and post-displacement azimuthal angle maps. If the displacement is known to be small a priori, we can restrict our search only to the closest neighbor singularities of the same topological charge. However, when the displacement is large and/or non-uniform and no information is given a priori, we cannot uniquely identify the corresponding singularities. To solve this problem, we make use of additional information about the core structure of the singularities in the azimuthal angle map. Similarly to optical vortices in random laser speckle fields ${ }^{19,20}$, the changes of the azimuthal angle around the singularities are nonuniform, and the typical core structure around the singularities are strongly anisotropic. Figure 1 shows an example of the amplitude contours of $\left|\vec{U}_{f}(x, y)\right|$ and neighborhood of a singularity; the azimuthal angle has a characteristic feature of a $2 \pi$ helical structure. The singularity is located at the center of the elliptical contours of the amplitude $\left|\vec{U}_{f}(x, y)\right|$, which is the intersection of the zero crossings of the two components $U_{f}^{(1)}(x, y)$ and $U_{f}^{(2)}(x, y)$ of the vector signal representation. We note that the eccentricity of the contour ellipse $e$ and the zero crossing angle $\theta_{\mathrm{RI}}$ between the $U_{f}^{(1)}$ and $U_{f}^{(2)}$, shown in Fig. 4(a), are invariant to the in-plane rigid-body motion of the object involving translation and rotation, and we use these two geometric parameters to describe the local properties of the phase singularities. In addition, each singularity has its own topological charge and vorticity defined by $\Omega=\nabla\left\{U_{f}^{(1)}(x, y)\right\} \times \nabla\left\{U_{f}^{(2)}(x, y)\right\}$, which we also assume to be invariant to the in-plane rigid-body displacement involving translation and rotation. Just as no fingers have exactly the same fingerprint patterns, no singularities have exactly the same local properties with identical eccentricity $e$, zero-crossing angle $\theta_{\mathrm{RI}}$, topological charge $q$, and vorticity $\Omega$. It is this uniqueness of the core structure that enables the correct identification and the tracking of the complicated movements of singularities. The matching between the pre- and post-displacement singularities are done on the basis of a merit function representing the degree of similarity between these parameters expressed by a distance in their parameter space.

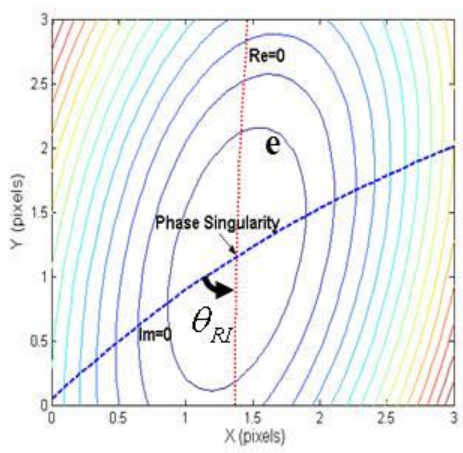

(a)

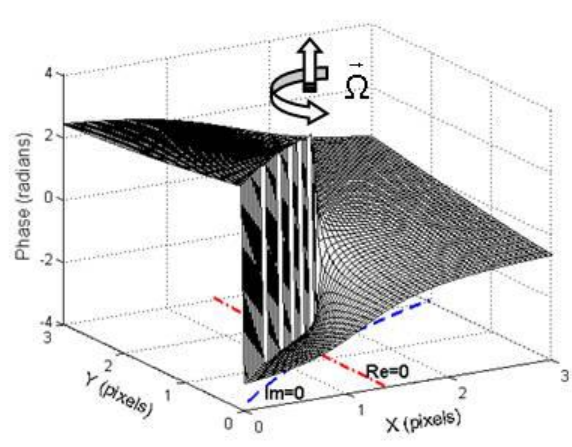

(b)

Figure 4. Core structure around a singularity for azimuthal angle $\varphi$. (a) Amplitude contours and zero crossing lines; (b) 3D- structure for singular azimuthal angle 
To measure lateral displacements in the micrometer range and rotational micro-displacements, we introduced controllable lateral and rotational micro-displacements with the precision mechanical stage of the microscope. From the recorded random texture patterns on the surface of the precision stage, we generated a vector signal representation by Riesz transform and retrieved the singularities information from the azimuthal angle map. In the experiment, we adjusted the average speckle size and controlled the density of singularities carefully by applying an additional low pass fileter with a proper bandwidth in the Fourier frequency domain, so that a single speckle includes about 40 pixels along a traversing line. After identifying the corresponding singularities for the object before and after displacement, we measured the given displacement by the proposed optical vortex metrology. Figure 5 (a) shows the parallel movement of singularities in the azimuthal angle map caused by the lateral displacement introduced by the translation stage. The displacements in the $x$ and $y$ directions were found to be $(\Delta x, \Delta y)=(19.6 \pm 0.4 \mu m, 21.2 \pm 0.3 \mu m)$. Figure 5 (b) shows the rotational movement of singularities caused by the rotational displacement introduced by the rotation stage. The rotation angle was found to be -518 milli-radians with the standard deviation 2milli-radians. We have also demonstrated optical vortex metrology for displacement measurement with nanometric resolution ${ }^{11,12}$, and for random flow measurements ${ }^{13}$.

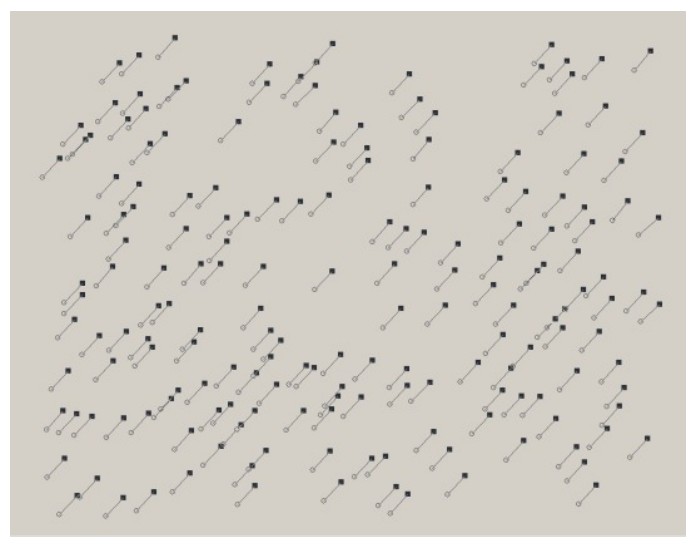

(a)

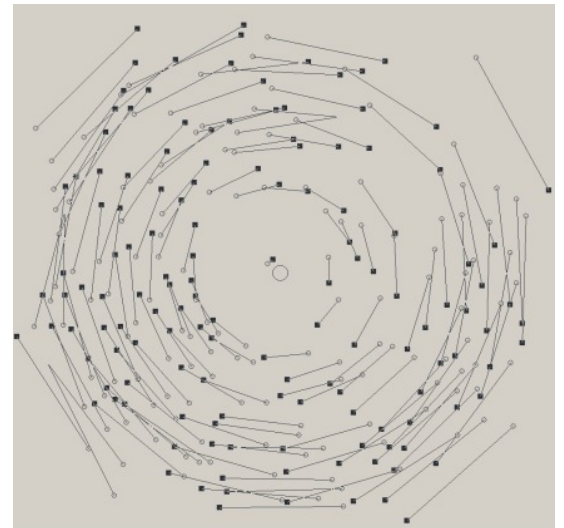

(b)

Figure 5. Movement of singularities in azimuthal angle caused by (a) lateral micro-displacement, and (b) rotational microdisplacement

In addition to the information about the anisotropic core structure for an individual singularity in azimuthal angle map as its unique fingerprint for reliable identification, we detected a group of optical vortices (singularity clusters) with specific mutual spatial structure to further strengthen an unambiguous tracking over the entire field of view ${ }^{14}$. To demonstrate the performance of the proposed technique, a swimming fugu fish with a speckle-like intensity pattern on its body surface, as seen in Fig.6 (a) and (b), was used as a biological specimen. First, a series of images of the swimming fugu fish were recorded by a high-speed camera, FASTCAM-NET 500/1000/Max (PHOTRON) with a pixel size of $7.4 \mu \mathrm{m} \times 7.4 \mu \mathrm{m}$. From the recorded images, we generated a vector signal by Riesz transform, and retrieved the azimuthal angle information. Similarly, we adjusted the average speckle size by choosing a proper bandwidth of the low pass filter when Riesz transforms were calculated through Fourier transform. In the experiment, we have controlled the density of the singularities carefully so that the singularities within the fugu body are clearly separated with a large average separation distance. Then, the constellation of singularities in azimuthal angle of the generated vector signal was identified and tracked during the specimen's motion across the entire field of view of the camera. After identifying corresponding singularities for each pair of consecutive images making use of their core structures as fingerprints, we traced the movement of the swimming fugu through its trajectory as shown in Fig. 6 (c) and (d). From the coordinate information for each singularity in the constellation, we conducted the in vivo measurement and obtain instant information for this swimming fugu about its translation, rotation, and scaling ${ }^{14}$. 

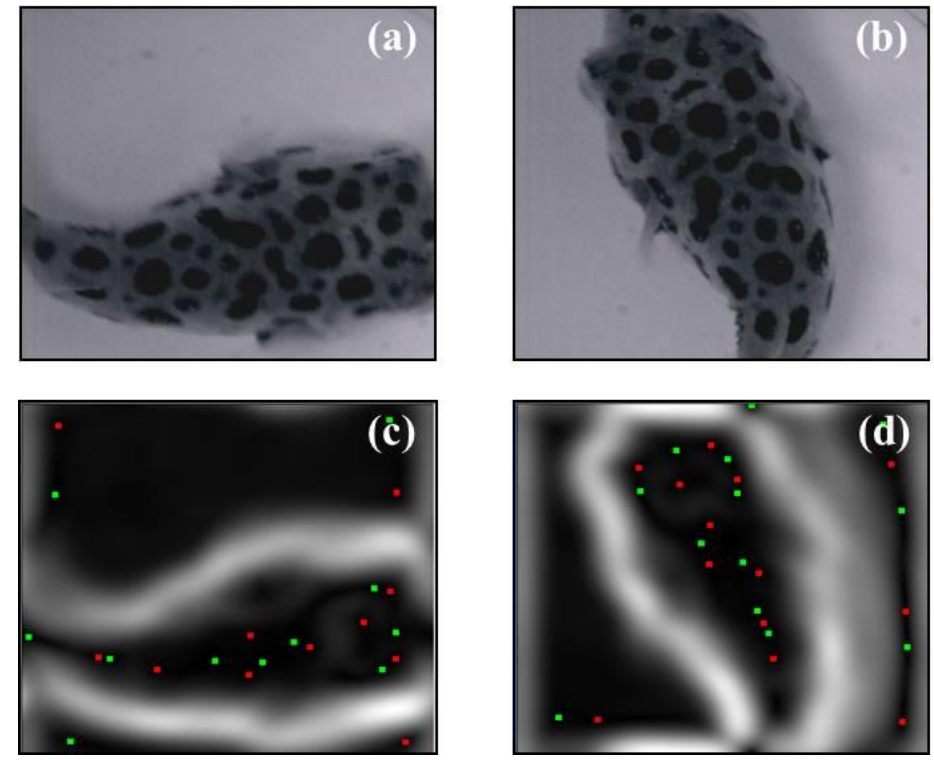

Figure 6. Recorded images for the swimming fugu and the corresponding azimuthal angle singularities: (a) and (c) are recorded at $\mathrm{t}=0.70$ seconds; $(\mathrm{b})$ and $(\mathrm{d})$ at $\mathrm{t}=3.33$ seconds.

\subsection{Pseudo-Stokes vector correlation}

Optical correlation techniques for measurements of random optical fields have constituted a promising area of optical information technology and have received a lot of interests. As an improvement of the intensity correlation used widely in conventional electronic speckle photography, recently, we proposed a new technique for in-plane displacement measurement, referred to as the Pseudo-Stokes Vector Correlation (PSVC) and demonstrated its performance superior to that of the conventional intensity-based correlation techniques ${ }^{15,16}$. The key idea of this technique is to use the pseudo Stokes vector information obtained from the isotropic vector signal representation generated from a Riesz transform of the intensity speckle pattern; therefore, no interferometer is required.

Let $I(x, y)$ be the AC component of the original intensity distribution of the speckle pattern obtained after subtraction of the mean value (average DC component). We can relate $I(x, y)$ to its associated complex signal in terms of a Riesz transform, i.e. $\tilde{I}(x, y)=U_{f}^{(1)}(x, y)+j U_{f}^{(2)}(x, y)$. Then a complex vector field $\nabla \tilde{I}(x, y)$, which is the associated gradient of the complex signal $\tilde{I}(x, y)$, can be expressed as

$$
\nabla \tilde{I}=\left(\partial_{x} U_{f}^{(1)}+j \partial_{x} U_{f}^{(2)}\right) x+\left(\partial_{y} U_{f}^{(1)}+j \partial_{y} U_{f}^{(2)}\right) y=\left(\partial_{x} U_{f}^{(1)} x+\partial_{y} U_{f}^{(1)} y\right)+j\left(\partial_{x} U_{f}^{(2)} x+j \partial_{y} U_{f}^{(2)} y\right) .
$$

Based on similar geometric features associated with the vector polarization field, a set of Stokes-like parameters has been proposed for the complex vector field $\nabla \tilde{I}^{21,22}$. Those are

$$
\begin{aligned}
& S_{0}(x, y)=\left(\partial_{x} U_{f}^{(1)}\right)^{2}+\left(\partial_{x} U_{f}^{(2)}\right)^{2}+\left(\partial_{y} U_{f}^{(1)}\right)^{2}+\left(\partial_{y} U_{f}^{(2)}\right)^{2}, \\
& S_{1}(x, y)=\left(\partial_{x} U_{f}^{(1)}\right)^{2}+\left(\partial_{x} U_{f}^{(2)}\right)^{2}-\left(\partial_{y} U_{f}^{(1)}\right)^{2}-\left(\partial_{y} U_{f}^{(2)}\right)^{2}, \\
& S_{2}(x, y)=2\left[\left(\partial_{x} U_{f}^{(1)}\right)\left(\partial_{y} U_{f}^{(1)}\right)+\left(\partial_{x} U_{f}^{(2)}\right)\left(\partial_{y} U_{f}^{(2)}\right)\right], \\
& S_{3}(x, y)=2\left[\left(\partial_{x} U_{f}^{(1)}\right)\left(\partial_{y} U_{f}^{(2)}\right)-\left(\partial_{x} U_{f}^{(2)}\right)\left(\partial_{y} U_{f}^{(1)}\right)\right] .
\end{aligned}
$$

The vector $\vec{S}(x, y)=\left[S_{1}, S_{2}, S_{3}\right]^{\dagger}$ is referred to as pseudo Stokes vector to distinguish it from the true Stokes parameter used for the polarization field of a vector wave. Thus, the proposed pseudo Stokes vector correlation (PSVC) can be defined as the inner correlation of pseudo Stoke vectors corresponding to the speckle pattern between the pre- and postdisplacement. That is

$$
C_{P S V C}(\Delta x, \Delta y)=\vec{S}(x, y) \oplus \vec{S}^{\prime}(x, y) .
$$


We conducted experiments to demonstrate the validity of the proposed technique. We generate the laser speckle pattern by illuminating an object with rough surface by He-Ne laser. The generated speckle pattern was imaged with a lens onto a CCD image sensor. By taking two images of the object before and after displacement, we measured the microdisplacement by the proposed pseudo Stokes vector correlation technique. To make the comparison on equal grounds, we calculated the correlation with a sub-window size of 100x100 pixels, and the shapes of the correlation functions for intensity correlation and PSVC are illustrated in Fig. 7(a). For both techniques, a main correlation peak can be observed at the coordinate $\Delta x=-13$ pixels, which indicates the displacement of the object. Near the main peak, we can also observe other small peaks, which can be a source of discrimination error in displacement measurement. The ratio of the main peak to its highest side-lobe can serve as a reliability measurement of the technique used. In this measure, the ratios of heights are 6.48 for IC and 7.02 for PSVC, respectively. By decreasing the size for the correlation window, we tested the performance of these two correlation techniques as shown in Fig. 7(b). As expected, the side lobes of the normalized correlation function increase with decreasing the correlation area. Unlike a small ratio equal to 1.62 given by the conventional IC technique, the proposed PSVC gives a very large ratio with 4.74, which indicates a strong robustness against decorrelation. In this case, a small sub-window of 30x30 pixels has been chosen for both methods. Figure 1 demonstrates the validity and advantage of the proposed pseudo Stokes vector correlation technique as compared with conventional intensity correlation.

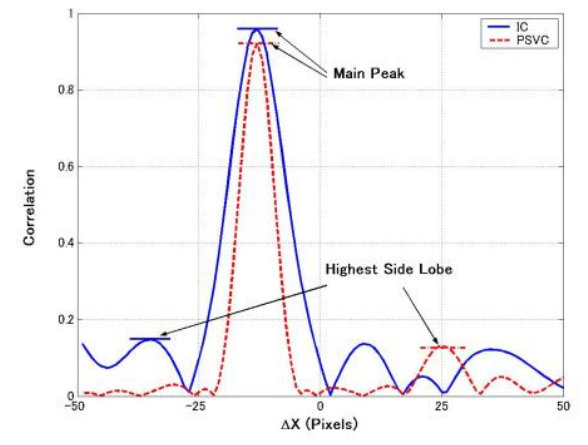

(a)

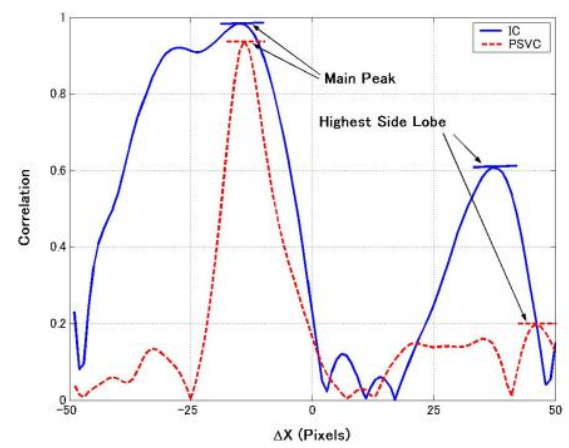

(b)

Figure 7. Distributions of correlation functions with different sub- window size (a) 100x100 pixels (b) 30x30 pixels

\section{CONCLUSIONS}

As a natural extension of 1-D analytic signal derived from Hilbert transform, we have introduced the vector signal representation of a 2-D speckle pattern as typical random real-valued signal generated from Riesz transform. We presented a number of interesting statistical properties similar to statistics of the 1-D analytic signal. We also reviewed several approaches proposed recently for translational and rotational displacement measurements that make use of the vector signal representation of a speckle pattern derived from Riesz filtering and provided experimental results to demonstrate the validity of the proposed techniques. Furthermore, the newly introduced modulus and angles information in the vector signal representation and the corresponding vector correlations introduce the new opportunities to explore other stochastic approaches in speckle metrology.

\section{REFERENCES}

1. Gabor, D., “Theory of communication,” J. Inst. Elect. Eng, 93, 429-457, (1946).

2. Hahn, S. L., [Hilbert Transforms in Signal Processing], Artech House, (1996).

3. Goodman, J. W., [Statistical Optics], Wiley-Interscience, New York, (2000).

4. Mandel, L., and Wolf, E., [Optical Coherence and Quantum Optics], Cambridge university press, (1995).

5. Felsberg, M., and Sommer, G., "The monogenic signal," IEEE Trans.Signal Process. 49, 3136-3144, (2001).

6. Hahn, S. L., "Multidimensional complex signals with single-orthant spectra," Proceedings of the IEEE, 80, 12871300, (1992). 
7. Felsberg, M., and Sommer, G., "A new extension of linear signal processingfor estimating local properties and detecting features," Proc. 22nd DAGM Symp. 195-202 (2000).

8. Larkin, K. G., Bone, J. D., and Oldfield, A. M., "Natural demodulation of two-dimensional fringe patterns. I. General background of the spiral phase quadrature transform," J. Opt. Soc. Am. A 18, 1862-1870, (2001).

9. Nabighian, M. N., "Toward a three-dimensional automatic interpretation of potential field data via generalized Hilbert transforms: Fundamental relations," Geophysics 49, 780-786, (1984).

10. Riesz, M., "Sur les fonctions conjuguées," Mathematische Zeitschrift 27, 218-244, (1928).

11. Wang, W., Yokozeki, T., Ishijima, R., Wada, A., Hanson, S. G., Miyamoto, Y., and Takeda, M., "Optical vortex metrology for nanometric speckle displacement measurement," Opt. Express 14, 120-127, (2006).

12. Wang, W., Yokozeki, T., Ishijima, R., Hanson, S. G., and Takeda, M., "Optical vortex metrology based on the core structures of phase singularities in Laguerre-Gauss transform of a speckle pattern," Opt. Express 14, 10195-10206, 2006.

13. Wang, W., Dennis, M. R., Ishijima, R., Yokozeki, T., Matsuda, A., Hanson, S. G., and Takeda, M., "Poincaré sphere representation for the anisotropy of phase singularities and its applications to optical vortex metrology for fluid mechanical analysis," Opt. Express, 15, 11008-11019, 2007.

14. Wang, W., Qiao, Y., Ishijima, R., Yokozeki, T., Honda, D., Matsuda, A., Hanson, S. G. and Takeda, M., "Constellation of phase singularities in a specklelike pattern for optical vortex metrology applied to biological kinematic analysis," Opt. Express,16, 13908-13917, 2008.

15. Wang, W., Ishijima, R. Matsuda, A., Hanson, S. G., and Takeda, M. "Pseudo-Stokes Vector Correlation From Complex Signal Representation of a Speckle Pattern and Its Applications to Micro-Displacement Measurement," Strain, 46, 12-18, (2010).

16. Wang, W., Zhang, S., Hanson, S. G., and Takeda, M., "Statistical properties of Derivatives of analytic signal generated by Riesz transform and its application to pesudo-Stokes vector correlation," Optics \& Photonics Japan 2009, Postdeadline Paper No.: 26pPD1 (2009).

17. Wang, W., Zhang, S., Ma, N., "Correlation properties of the vector signal representation for speckle pattern," Appl. Opt. 53(10), B147-B152, (2014).

18. Bracewell, R. N., [The Fourier transform and its applications], 3rd Edition, McGraw-Hill, (1999).

19. Berry, M. V., and Dennis, M. R., "Phase singularities in isotropic random waves," Proc. R. Soc. Lond. A, 456, 2059-2079, (2000).

20. Wang, W., Hanson, S. G., Miyamoto, Y., and Takeda, M., "Experimental investigation of local properties and statistics of optical vortices in random wave fields," Phys. Rev. Lett. 94, 103902, (2005).

21. Dennis, M. R., "Local structure of wave dislocation lines: twist and twirl," J. Opt. A: Pure Appl. Opt. 6, s202-s208 (2004).

22. Egorov, Y. A., Fadeyeva, T. A., and Volyar, A. V., "The fine structure of singular beams in crystals: colours and polarization,” J. Opt. A: Pure Appl. Opt. 6, s217-s228 (2004). 